\title{
Estudo da ocorrência de infecção hospitalar em cães e gatos em um centro cirúrgico veterinário universitário ${ }^{1}$
}

\author{
Mônica V. Bahr Arias²*, Graciane Aiello ${ }^{3}$, Luana de A. Battaglia ${ }^{4}$ e Julio C. de Freitas ${ }^{5}$
}

\begin{abstract}
Bahr Arias M.V., Aiello G., Battaglia L.A. \& Freitas J.C. 2013. [Study of nosocomial infections in dogs and cats in an academic veterinary surgical center.] Estudo da ocorrência de infecção hospitalar em cães e gatos em um centro cirúrgico veterinário universitário. Pesquisa Veterinária Brasileira 33(6):771-779. Departamento de Clínicas Veterinárias, Universidade Estadual de Londrina, Rodovia Celso Garcia Cid, PR $445 \mathrm{Km} 380$, Campus Universitário, Cx. Postal 10011, Londrina, PR 86057-970, Brazil. E-mail: vicky@uel.br

Hospital or nosocomial infections are infections acquired during patient hospitalization, and that can be related to surgical and invasive procedures performed during the hospital admission. The present study aimed to investigate the occurrence of nosocomial infection in animals treated at a Veterinary Surgical Center of Small Animals, submitted to surgical or invasive procedures, to discuss the possible causes of infection, to detect the bacteria present when possible, and to verify the antimicrobial sensitivity of these agents. The study was developed through daily monitoring of 131 animals admitted in this surgical center and doing active surveillance of cases of nosocomial infection. In 104 animals (91 dogs and 13 cats), 113 surgical procedures were performed, and in 27 animals were performed non-surgical procedures such monitoring of delivery and postpartum, urethral catheterization and placement of splints. All animals were submitted to catheter placement for fluid therapy and application of medications and/or anesthetic at some point during hospitalization. The rate of surgical site infection was $7.96 \%$, and by categories was $4.54 \%$ in clean surgeries, $4.25 \%$ in clean-contaminated surgeries, $10.53 \%$ in contaminated surgeries, and $16 \%$ in infected surgeries. The rate of non-surgical nosocomial infection in surgical patients was $2.88 \%$ and in the non-surgical patient was 3.7\%. Bacteria were cultured as follows: Pseudomonas sp. (3), Streptococcus sp. (2), Acinetobacter sp. (1) and Gram negative bacilli (1), and high bacterial multidrug resistance were observed in all isolates. The duration of surgery and pre and postoperative time of hospitalization did not affect the occurrence of nosocomial infection, but factors that probably contributed to the occurrence of infections in this study were the severity of the condition responsible for the treatment, the kind of procedure performed and the severity of lesions.

INDEX TERMS: Nosocomial infections, infectious disease, antimicrobial resistance, bacteriology, dogs, cats.
\end{abstract}

\footnotetext{
${ }^{1}$ Recebido em 5 de dezembro de 2012.

Aceito para publicação em 24 de janeiro de 2013.

${ }^{2}$ Docente do Departamento de Clínicas Veterinárias, Universidade Estadual de Londrina (UEL), Rodov. Celso Garcia Cid, PR $445 \mathrm{Km}$ 380, Campus Universitário, Cx. Postal 10011, Londrina, PR 86057-970, Brasil. *Autor para correspondência: vicky@uel.br

${ }^{3}$ Doutoranda em Cirurgia Veterinária, Universidade Federal de Santa Maria, Hospital Veterinário, prédio 97, sala 126, Av. Roraima 1000, Campus Universitário, Santa Maria, RS 97105-900, Brasil.

${ }^{4}$ Médica Veterinária Autônoma, Clínica Geral e Intensivista do Hospital Veterinário Sena Madureira, Av. Sena Madureira 898, São Paulo, SP 04021001, e Membro da equipe de Neurologia do Hospital Veterinário PetCare Pacaembu, Av. Pacaembu 1839, São Paulo, SP, 01234-001, Brasil.

${ }^{5}$ Docente, Departamento de Medicina Veterinária Preventiva, UEL, Cx. Postal 10011, Londrina, PR 86057-970.
}

RESUMO-- Infecção hospitalar ou nosocomial é aquela adquirida durante a hospitalização do paciente, e que pode ser relacionada os procedimentos hospitalares invasivos realizados durante o internamento. 0 presente trabalho teve como objetivos estudar a ocorrência de infecção hospitalar em animais atendidos em um Centro Cirúrgico Veterinário Universitário de Pequenos Animais submetidos a procedimentos cirúrgicos e/ou invasivos; discutir as possíveis causas de infecção, detectar as bactérias presentes quando possível e verificar a sensibilidade antimicrobiana destes agentes. 0 trabalho foi desenvolvido através do acompanhamento diário de 131 animais internados neste setor e busca ativa de casos de infecção hospitalar. Em 104 animais 
(91 cães e 13 felinos), foram realizados 113 procedimentos cirúrgicos e em 27 animais condutas não cirúrgicas tais como acompanhamento de parto e pós-parto, desobstrução uretral e colocação de talas. Todos os animais foram submetidos à colocação de cateter para fluidoterapia e/ ou aplicação de medicamentos e/ou anestésicos em algum momento durante o internamento. 0 índice de infecção do sítio cirúrgico foi de 7,96\% sendo 4,54\% nas cirurgias limpas, $4,25 \%$ nas cirurgias limpa-contaminadas, $10,53 \%$ nas cirurgias contaminadas e $16 \%$ nas cirurgias infectadas. A taxa de infecção hospitalar não cirúrgica no paciente cirúrgico foi de $2,88 \%$ e $3,7 \%$ no paciente não cirúrgico. Foram cultivados sete isolados bacterianos, sendo Pseudomonas sp. (3), Streptococcus sp. (2), Acinetobacter sp. (1) e bacilo Gram negativo (1), constatando-se multirresistência bacteriana alta em todos os isolados. A duração da cirurgia e os tempos de internamento pré e pós-operatório não influenciaram na ocorrência de infecção hospitalar, mas os fatores que provavelmente colaboraram para a ocorrência de infecções no presente trabalho foram a própria gravidade da doença que motivou o tratamento, o tipo de procedimento realizado e a gravidade das lesões concomitantes.

TERMOS DE INDEXAÇÃO: Infecção hospitalar, doenças infecciosas, resistência antimicrobiana, bacteriologia, cães, gatos.

\section{INTRODUÇÃO}

Infecções hospitalares ou nosocomiais são infecções adquiridas após a hospitalização do paciente, que se manifestam durante a internação ou mesmo após a alta, e estão relacionadas com a internação ou procedimentos hospitalares realizados (Fernandes et al. 2000, Santos et al. 2012), causadas por bactérias ou outros organismos infecciosos (Johnson 2002). As bactérias associadas a estas infecções são frequentemente resistentes aos antimicrobianos usados rotineiramente (Johnson 2002, Santos et al. 2012). Infecções hospitalares acometem cinco a 10\% dos pacientes humanos hospitalizados, afetando o bem estar do paciente, causando aumento da morbidade, mortalidade e custos (Johnson 2002). As principais síndromes hospitalares que acometem o paciente humano internado são as infecções da corrente sanguínea, infecção do sítio cirúrgico, infecção urinária, infecção respiratória e sepse (Lacerda 1992, Ogeer-Gyles et al. 2006, Johnson 2002).

A incidência da infecção hospitalar não está bem estabelecida em Medicina Veterinária principalmente devido à falta de Comissões de Controle de Infecção Hospitalar (Braga 2008, Santos et al. 2012), mas os fatores que contribuem para sua ocorrência em humanos também estão se tornando mais comuns nos animais, devido ao aumento da quantidade e qualidade de cuidados intensivos realizados, ao uso de dispositivos intravasculares e sondas vesicais, aumento do tempo de hospitalização, uso indiscriminado de antimicrobianos, realização de cirurgias com colocação de implantes e uso de drogas imunossupressoras (Johnson 2002, Nicholson et al. 2002, Sanchez et al. 2002, Eugster et al. 2004, Ogeer-Gyles et al. 2006, Marsh-ng et al. 2007, Pelligand et al. 2007, Weese 2012). 0 paciente cirúrgico durante os períodos pré, trans e pós-operatório está sujeito à realização de vários procedimentos invasivos além da cirurgia propriamente dita, que podem favorecer a ocorrência de infecção hospitalar, repercutindo negativamente em sua recuperação, inclusive podendo causar óbito (Lacerda 1992, Johnson 2002, Greene \& Dearmin 2006, Santos et al. 2012). Outros aspectos importantes quando da ocorrência de infecção hospitalar são a insatisfação do proprietário, as implicações ao profissional responsável pelo paciente e os fatores econômicos devido ao aumento dos custos do tratamento (Brown 2012, Weese 2012).

A infecção hospitalar pode ter origem endógena, devido à doença ou condição clínica predisponente e ao tipo de cirurgia realizada, ou exógena, causada por contaminação de artigos médicos hospitalares, do ambiente, equipe e material cirúrgico (Lacerda 1992, Brown 2012). É importante lembrar que contaminação é a presença transitória de micro-organismos em superfície sem invasão tecidual, que pode ocorrer em objetos inanimados ou em hospedeiros, enquanto que infecção são os danos decorrentes da multiplicação, ação destes patógenos e/ou de seus produtos tóxicos no hospedeiro, ocorrendo interação imunológica (Fernandes et al. 2000). Embora a contaminação de superfícies tenha importância no estudo da infecção, não é o único fator envolvido (Lacerda 1992). Vários autores focaram os estudos de infecção hospitalar na microbiota do ar, superfícies (Costa et al. 1996, Mendes et al. 2005, Santos et al. 2010) e pessoal envolvido no atendimento dos pacientes veterinários (Andrade et al. 1992), considerando a infecção hospitalar consequência apenas de falhas da desinfecção de ambientes, ocorrendo a disseminação de medidas impróprias e ineficazes na sua prevenção, em detrimento de outras condutas importantes (Lacerda 1992, Weese 2012). O Center for Disease Control (CDC) preconiza que um programa de controle de infecção adequado não deve realizar a vigilância do ambiente e identificação das bactérias do ar (CDC 2008), pois o ambiente nunca foi nem será um meio estéril e esta conduta leva a desperdício de tempo e dinheiro e identificação de micro-organismos que dificilmente estão relacionados ao contexto da infecção (Weese 2012).

0 risco de infecção correlaciona-se com a quantidade de inóculo microbiano, a sua virulência e varia inversamente com a resistência do paciente (Nicholson et al. 2002). A classificação das feridas cirúrgicas é usada em medicina humana desde 1964 (Greene \& Dearmin 2006) para reconhecer as taxas de infecção, permitindo uma análise comparativa dos dados e a adoção de medidas de controle e prevenção (Lacerda 1992). Segundo Fossum \& Willard (2007), embora a classificação da ferida cirúrgica seja útil para prever a possibilidade de ocorrência de infecção, pode haver sobreposição e inconsistência na divisão de vários grupos. Já para Dunning (2003), este sistema é útil para orientar o uso de antimicrobianos, o manejo do paciente e a avaliação pré e pós-operatória dos casos. Assim, o Sistema Nacional Americano de Pesquisa em Infecções Nosocomiais (NNISS) propôs que não só a classificação da ferida cirúrgica quanto ao potencial de contaminação deva ser considerado para prever o risco de ocorrência de infecção do sítio cirúrgico, mas mais dois aspectos: o estado prévio do paciente segundo a Sociedade Americana de Anestesiologia (ASA) e a du- 
Quadro 1. Classificação das feridas cirúrgicas quanto ao potencial de contaminação, descrição, exemplos e taxas descritas em literatura

\begin{tabular}{|c|c|c|c|}
\hline Classificação & Descrição & Exemplos & $\begin{array}{l}\text { Variação das taxas de in- } \\
\text { fecção de sítio cirúrgico } \\
\text { em cães e gatos }\end{array}$ \\
\hline Limpa & $\begin{array}{l}\text { Realizadas em tecidos estéreis } \\
\text { Não traumática } \\
\text { Não há inflamação ou infecção } \\
\text { Sem penetração em superfícies mucosas, ou seja, } \\
\text { não há entrada em trato respiratório, alimentar, bi- } \\
\text { liar ou genitourinário } \\
\text { Sem falha de técnica }\end{array}$ & $\begin{array}{l}\text { Cirurgias Neurológicas } \\
\text { Herniorrafias (inguinal, umbilical) } \\
\text { Biópsias excisionais cutâneas sem presença de ul- } \\
\text { ceração } \\
\text { Mastectomia sem ovariosalpingohisterectomia (OSH) } \\
\text { Cirurgias ortopédicas não traumáticas (correção de } \\
\text { luxação de patela e ruptura de ligamento cruzado) } \\
\text { Cirurgia eletiva - oriquiectomia }\end{array}$ & $0-5,5 \%$ \\
\hline Limpo- contaminada & $\begin{array}{l}\text { Penetração na microbiota endógena sob controle: } \\
\text { penetração na luz de órgãos do trato respiratório, } \\
\text { digestivo, genital ou urinário, sem extravasamento } \\
\text { significativo de conteúdo, sem contaminação exter- } \\
\text { na, sem inflamação supurativa } \\
\text { Quebra menor da assepsia (perfuração em luva). } \\
\text { Cirurgias limpas com duração superior a } 3 \text { horas. }\end{array}$ & $\begin{array}{l}\text { Cirurgia gástrica, enterotomia, cistotomia, hernior- } \\
\text { rafia perineal, otohematoma, OSH eletiva } \\
\text { Cirurgias oftálmicas } \\
\text { Cirurgias ortopédicas decorrentes de trauma (fra- } \\
\text { turas não expostas) }\end{array}$ & $3,5-10 \%$ \\
\hline Infectada & $\begin{array}{l}\text { Cirurgias realizada na presença de pus, processo in- } \\
\text { feccioso coexistente, tecido desvitalizado ou víscera } \\
\text { perfurada } \\
\text { Quebra maior na assepsia }\end{array}$ & $\begin{array}{l}\text { OSH para tratamento de piometra ou morte fetal, vís- } \\
\text { ceras previamente perfuradas, correção de fraturas } \\
\text { expostas com fragmento avascular, tecido necrótico, } \\
\text { secreção purulenta }\end{array}$ & $6,7-40 \%$ \\
\hline
\end{tabular}

Referências: Dunning 2003, Eugster et al. 2004, Greene \& Dearmin 2006, CDC 2008, Brown 2012.

Abreviatura: $\mathrm{OSH}=$ ovariosalpingohisterectomia.

ração da cirurgia. O NNIS desenvolveu um índice baseado nestes três fatores, e o resultado foi comparado com os resultados obtidos pela classificação da ferida cirúrgica. Este estudo concluiu que a utilização dos três fatores mostra melhor a realidade dos riscos (Greene \& Dearmin 2006).

0 monitoramento da ocorrência de infecção hospitalar em veterinária, apesar de extremamente importante, não tem sido valorizado (Ogeer-Gyles et al. 2006). Assim, os objetivos do presente trabalho foram estudar a ocorrência de infecção hospitalar em animais internados em um Centro Cirúrgico Veterinário Universitário de Pequenos Animais submetidos a procedimentos cirúrgicos e/ou invasivos; discutir as possíveis causas de infecção, detectar as bactérias presentes, quando possível, e verificar a susceptibilidade destas cepas frente aos antibacterianos usados neste hospital.

\section{MATERIAL E MÉTODOS}

Este trabalho foi realizado no Setor de internamento do Centro Cirúrgico do Hospital Veterinário da Universidade Estadual de Londrina, entre 09/2006 e 04/2007. 0 trabalho foi desenvolvido por meio do acompanhamento diário de 131 animais internados neste setor e busca ativa de casos de infecção hospitalar. Em 104 animais (91 cães e 13 gatos), foram realizados 113 procedimentos cirúrgicos e em 27 animais condutas não cirúrgicas tais como acompanhamento de parto e pós-parto, desobstrução uretral e colocação de talas. Todos os animais foram submetidos à colocação de cateter para fluidoterapia e/ou aplicação de medicamentos e/ou anestésicos em algum momento durante o internamento.

Os animais foram individualmente acompanhados preen- chendo-se uma ficha no qual constava resenha, motivo do internamento, diagnóstico, procedimento cirúrgico realizado, produtos utilizados na anti-sepsia, administração de antibiótico profilático, risco cirúrgico, medicamentos utilizados no pós-operatório (dose, intervalo e duração), procedimentos invasivos (cateter venoso, sondas vesicais), alterações clínicas encontradas no pós-operatório, resultados do antibiograma e antibioticoterapia instituída.

As cirurgias foram classificadas de acordo com o potencial de contaminação da ferida cirúrgica em cirurgias limpas, limpa-contaminadas, contaminadas e infectadas (Quadro 1) (Dunning 2003, Eugster et al. 2004, Greene \& Dearmin 2006, CDC 2008, Brown 2012). 0 estado físico geral do paciente foi classificado segundo a ASA (Greene \& Dearmin 2006) (Quadro 2). Foi considerado procedimento cirúrgico aquele que envolvesse a realização de incisão

\section{Quadro 2. Determinação do risco cirúrgico relacionado ao} estado físico pré-operatório do paciente (ASA)

\begin{tabular}{|c|c|c|}
\hline Risco & Condições do paciente & Exemplos \\
\hline I & $\begin{array}{l}\text { Saudável, sem doença reconhe- } \\
\text { cida }\end{array}$ & Procedimentos Eletivos \\
\hline II & $\begin{array}{l}\text { Saudável, com doença localizada, } \\
\text { sem sinais sistêmicos }\end{array}$ & $\begin{array}{l}\text { Luxação de patela; neoplasia cu- } \\
\text { tânea }\end{array}$ \\
\hline III & $\begin{array}{l}\text { Sinais sistêmicos moderados que } \\
\text { limitam a função }\end{array}$ & $\begin{array}{l}\text { Pneumonia, febre, desidratação, } \\
\text { anemia, sopro cardíaco }\end{array}$ \\
\hline IV & $\begin{array}{l}\text { Doença sistêmica grave com ris- } \\
\text { co de vida }\end{array}$ & $\begin{array}{l}\text { Insuficiência cardíaca, renal ou } \\
\text { hepática; hemorragia ou desidra- } \\
\text { tação severa }\end{array}$ \\
\hline V & $\begin{array}{l}\text { Moribundos, sem expectativa de } \\
\text { vida por mais de } 24 \text { horas com ou } \\
\text { sem cirurgia }\end{array}$ & $\begin{array}{l}\text { Choque endotóxico, falência mú- } \\
\text { ltipla de órgãos, trauma grave }\end{array}$ \\
\hline
\end{tabular}

Abreviatura: estado prévio do paciente segundo a Sociedade Americana de Anestesiologia. Referências: Greene \& Dearmin 2006. 
e sutura, assim, síntese de ferida traumática e drenagem de abscesso não foram considerados no presente estudo (Brasil 2009).

Para o diagnóstico de infecção de sítio cirúrgico (ISC), considerou-se: 1) ocorrência de secreção purulenta na incisão superficial (com ou sem confirmação laboratorial) desde que a secreção purulenta não caracterizasse reação local ao ponto; 2) ocorrência de deiscência espontânea dos pontos ou micro-organismos isolados de uma cultura obtida assepticamente de secreção obtida da incisão. A infecção foi classificada como: superficial (se localizada acima da fáscia muscular), profunda (se localizada entre a fáscia e a camada muscular), e órgão/cavidade (quando envolvesse estruturas anatômicas, abertas ou manipuladas, durante o ato cirúrgico) (Mangram 1999, Brasil 2009, Weese 2012). A taxa de infecção do sítio cirúrgico foi calculada considerando-se o numerador o número de infecções cirúrgicas e o denominador o número de procedimentos realizados, sendo a razão multiplicada por 100 e expressa em porcentagem (Brasil 2009). Considerou-se ocorrência de infecção hospitalar não cirúrgica a infecção relacionada ao cateter venoso, à sonda vesical ou ocorrência de sepse, sendo a taxa calculada em relação ao número de pacientes (Fernandes et al. 2000).

Em sete pacientes com sinais de infecção foi realizada a coleta de secreção com suabe estéril para realização de cultura bac- teriana. Após a identificação dos micro-organismos envolvidos nos processos infecciosos, as amostras foram testadas quanto à susceptibilidade a antimicrobianos utilizando-se o método de difusão em disco com antimicrobianos geralmente indicados na terapêutica para cada sítio de infecção. As zonas de inibição dos antimicrobianos foram interpretados de acordo com Clinical and Laboratory Standards Institute (CLSI 2008). Considerou-se multirresistência a resistência a mais de duas classes de antimicrobianos (CDC 2008).

Os resultados foram dispostos em frequência e porcentagem dentro de cada classe de pacientes e de cirurgia por potencial de contaminação, procurando-se avaliar as possíveis causas de infecção. As variáveis tempo operatório e de internamento foram dispostas em mínimo, máximo, média e mediana. 0 teste $t$ foi utilizado $(p<0,05)$ para avaliar se houve influência da duração da cirurgia e do tempo de internamento prévio e pós-operatório na ocorrência de infecção

\section{RESULTADOS}

Dos 131 animais acompanhados, em 104 foram realizados 113 procedimentos cirúrgicos, alguns simultaneamente no

Quadro 3. Número de animais por classe de cirurgia, tempo de duração do procedimento cirúrgico, tempo de internamento no período pré e pós-operatório

\begin{tabular}{|c|c|c|c|c|c|}
\hline & Limpa & Limpa-contaminada & Contaminada & Infectada & Total \\
\hline Cães & 17 & 38 & 17 & 19 & 91 \\
\hline Machos & 8 & 13 & 6 & 4 & 31 \\
\hline Fêmeas & 9 & 25 & 11 & 15 & 60 \\
\hline Gatos & 1 & 7 & 0 & 5 & 13 \\
\hline Machos & 1 & 3 & 0 & 1 & 5 \\
\hline Fêmeas & 0 & 4 & 0 & 4 & 8 \\
\hline \multicolumn{6}{|c|}{ Tempo operatório } \\
\hline Min - máx & 40 a $180 \mathrm{~min}$ & 40 a $300 \mathrm{~min}$ & 30 a $220 \mathrm{~min}$ & 30 a $210 \mathrm{~min}$ & $30-300 \mathrm{~min}$ \\
\hline Média & $119 \min$ & $129 \mathrm{~min}$ & $101 \mathrm{~min}$ & $86 \mathrm{~min}$ & 112 \\
\hline Mediana & 120 & 120 & 80 & 65 & 107 \\
\hline \multicolumn{6}{|c|}{ Tempo internamento pré-operatório } \\
\hline Min - max & 30 minutos a 3 dias & 30 minutos a 6 dias & 30 min a 4 dias & $60 \mathrm{~min}$ a 12 dias & $30 \mathrm{~min}$ a 12 dias \\
\hline Média & 1 dia & 2 dias & 1,2 dias & 1,1 dia & 1,5 dias \\
\hline Mediana & 6 horas & 2 dias & 1,1 dia & 1,1 dia & 14 horas \\
\hline \multicolumn{6}{|c|}{ Tempo internamento pós-operatório } \\
\hline Min - máx & 3 horas a 18 dias & 8 horas a 30 dias & 2 horas a 10 dias & 1 a 16 dias & 2 horas a 30 dias \\
\hline Média & 3 dias & 4 dias & 2 dias & 4 dias & 3,5 dias \\
\hline Mediana & 1,5 dias & 1 dia & 1 dia & 1,5 dias & 1 dia \\
\hline
\end{tabular}

Quadro 4. Número de animais e de cirurgias por classe de cirurgia, casos com sinais de infecção do sítio cirúrgico e de infecção hospitalar não cirúrgica dentro de cada classe, ASA dentro de cada classe, uso de antimicrobianos em 104 pacientes cirúrgicos

\begin{tabular}{|c|c|c|c|c|c|c|c|c|c|c|}
\hline \multirow[t]{2}{*}{ Classe das cirurgias } & \multicolumn{2}{|c|}{ Limpa } & \multicolumn{2}{|c|}{ Limpa-contaminada } & \multicolumn{2}{|c|}{ Contaminada } & \multicolumn{2}{|c|}{ Infectada } & \multicolumn{2}{|c|}{ Total } \\
\hline & $\mathrm{N}$ & $\%$ & $\mathrm{~N}$ & $\%$ & $\mathrm{~N}$ & $\%$ & $\mathrm{~N}$ & $\%$ & $\mathrm{~N}$ & $\%$ \\
\hline Número de animais & 18 & 17,3 & 45 & 43,27 & 17 & 16,35 & 24 & 23,07 & 104 & 100 \\
\hline Número de cirurgias & 22 & 19,46 & 47 & 41,6 & 19 & 16,8 & 25 & 22,1 & 113 & 100 \\
\hline ISC & 1 & 4,54 & 2 & 4,25 & 2 & 10,53 & 4 & 16 & 9 & 7,96 \\
\hline \multicolumn{11}{|l|}{ ASA (número de pacientes) } \\
\hline I & - & - & - & - & - & - & - & - & - & 0 \\
\hline II & 18 & 17,3 & 34 & 32,7 & 13 & 12,5 & 7 & 6,73 & 72 & 69,23 \\
\hline III & - & - & 11 & 10,57 & 4 & 3,84 & 13 & 12,5 & 28 & 26,92 \\
\hline IV & - & - & - & - & - & - & 4 & 3,84 & 4 & 3,84 \\
\hline Casos com sinais IH não cirúrgica & 0 & 0 & 3 & 6,67 & 0 & 0 & 0 & 0 & 3 & 2,88 \\
\hline Trauma Prévio & 4 & 3,84 & 20 & 19,23 & 9 & 8,65 & 5 & 4,81 & 38 & 36,5 \\
\hline Tratamento antimicrobiano prévio & 0 & 0 & 5 & 4,81 & 3 & 2,88 & 4 & 3,84 & 12 & 11,53 \\
\hline Profilaxia antimicrobiana & 8 & 7,69 & 21 & 46,67 & 7 & 6,73 & 9 & 8,65 & 45 & 43,27 \\
\hline Antimicrobiano Pós-operatório & 18 & 17,3 & 45 & 43,27 & 17 & 16,35 & 24 & 23,08 & 100 & 100 \\
\hline Implante ortopédico & 1 & 0,96 & 18 & 17,31 & 6 & 5,77 & 0 & 0 & 25 & 24,04 \\
\hline
\end{tabular}

Abreviaturas: ASA= estado prévio do paciente segundo a Sociedade Americana de Anestesiologia, que varia de I a V

(Quadro 2); IH = infecção hospitalar. 
mesmo animal. Foram operados 91 cães e 13 gatos (Quadro 3). A distribuição dos pacientes segundo a classe de potencial de contaminação da ferida cirúrgica, número de pacientes com sinais de infecção do sítio cirúrgico por classe e número de pacientes cirúrgicos com sinais de infecção decorrente de procedimentos invasivos não cirúrgicos estão no Quadro 4. Dentre os 27 casos não cirúrgicos constatou-se infecção urinária em um paciente felino $(3,7 \%)$, após sondagem vesical.

No Quadro 5 estão discriminados a espécie, raça, idade e sexo dos animais que apresentaram complicações da cirurgia ou dos procedimentos de apoio, o diagnóstico e alterações clínicas iniciais, o procedimento realizado, tempo de duração da cirurgia, estado físico prévio, alteração observada, a bactéria determinada na cultura e o tempo de internamento no HV até a realização da cirurgia.

Considerando todos os procedimentos cirúrgicos realizados, o tempo transoperatório médio foi de 112 minutos, não havendo pelo teste $\mathrm{T}$ de student diferença significativa entre tempo operatório e ocorrência de infecção nas diferentes classes (Quadro 3). 0 tempo de internamento pré-operatório variou entre 30 minutos e 12 dias e o tempo de internamento pós-operatório variou de duas horas a 30 dias (Quadro 3), não havendo pelo teste t de student diferença significativa entre estes valores.

Os resultados do antibiograma dos pacientes encontram-se no Quadro 6. Constatou-se multirresistência bacteriana em todos os isolados (Quadro 6).

Dentre os procedimentos não cirúrgicos, 12 animais foram submetidos à desobstrução uretral com sonda, 12 animais foram internados para colocação de talas devido a algum tipo de fratura e três animais foram internados para acompanhamento do parto. Um felino (1,35\%), submetido à desobstrução uretral necessitou permanecer com sonda uretral por cinco dias, recebendo enrofloxacina enquanto sondado. Na urocultura houve o crescimento de Acinetobacter sp. multirresistente (Quadro 6).

Constatou-se que 69,23\% dos pacientes não apresentavam sinais sistêmicos no momento da cirurgia (ASA II), enquanto que $30,76 \%$ apresentavam sinais sistêmicos moderados ou graves (ASA III e IV). Quatro casos de infecção hospitalar ocorreram em pacientes ASA II, seis em ASA III e dois em ASA IV (Quadro 5). Em 36,5\% dos pacientes houve trauma prévio como motivo para a realização da cirurgia, sendo que seis destes pacientes apresentaram infecção hospitalar. Em 11,53\% dos pacientes houve a necessidade de iniciar antibioticoterapia previamente à cirurgia. A administração de antimicrobianos no momento da indução anestésica foi realizada em $43,27 \%$ dos pacientes, enquanto que em todos houve seguimento da administração desta medicação.

\section{DISCUSSÃO}

No presente trabalho foi realizado o acompanhamento dos animais internados para realização de cirurgias ou de procedimentos não cirúrgicos, visando o diagnóstico de infecção hospitalar. É importante frisar que infecção hospitalar no paciente da ala cirúrgica não significa somente infecção do sítio cirúrgico (Lacerda 1992). Entretanto, ISC é o tipo de infecção mais estudada na Medicina Veterinária de animais de companhia (Bernis Filho et al. 1998, Johnson 2002, Eugster 2004, Braga 2008, Brown 2012). Nos pacientes internados para procedimentos cirúrgicos e submetidos a procedimentos invasivos, deve-se monitorar a ocorrência de infecção hospitalar, pois os procedimentos de apoio são causas importantes de infecção (Lacerda 1992), como constatado no presente estudo, onde $2,88 \%$ dos pacientes cirúrgicos e 3,7\% dos pacientes não cirúrgicos apresentaram infecção decorrente de procedimentos invasivos não cirúrgicos.

O acompanhamento dos casos foi realizado durante sete meses, tendo sido realizado pelo processo de busca ativa, conforme relatado por Braga (2008). É um método que identifica os pacientes acometidos por infecção hospitalar independentemente de sua notificação, sendo citada como a mais eficiente, pois envolve o contato direto com o paciente, a avaliação do resultado dos exames laboratoriais, do tempo de internamento, do prontuário, do uso de antimicrobianos e avaliação da ferida cirúrgica, porém conforme constatado por Eugster et al. (2004), Braga (2008) e pelos autores do presente estudo, é uma prática trabalhosa devido à falta de uma equipe designada e de padronização dos prontuários.

No Brasil, em medicina veterinária não existem muitos trabalhos sobre infecção hospitalar. Bernis Filho et al. (1998) avaliaram a infecção da ferida cirúrgica em pacientes caninos e felinos submetidos à cirurgias ortopédicas e não ortopédicas na UFMG, observando uma taxa de infecção de $9 \%$, havendo relação entre a duração do procedimento e ocorrência de infecção, conforme também observado por Nicholson et al. (2002) e Eugster et al. (2004), discordando dos resultados do presente estudo. Braga (2008) constatou uma incidência global de 9,5\%, acima do constatado no presente estudo, sendo de $10,4 \%$ na classe de cirurgias limpas e de 7,4\% na classe de contaminadas. Já Nicholson et al. (2002), em estudo realizado na Philadelphia, constataram uma taxa de infecção de 5,9\% na classe de cirurgias limpa contaminadas, levemente maior do que o observado no presente estudo, verificando que a duração da cirurgia e a presença de endocrinopatias estavam relacionadas à ocorrência de infecção. Segundo Braga (2008), a comparação entre taxas de infecção deve ser vista com cautela, pois existem muitas variáveis como o método de vigilância empregado, as doenças que motivaram o internamento, o uso de antimicrobianos e o grau de complexidade dos procedimentos realizados, entre outros fatores.

Na classe das cirurgias limpas, o índice de 4,54 está dentro dos valores considerados aceitáveis. É importante lembrar que outros fatores, além da infecção, podem colaborar para a deiscência dos pontos. A técnica cirúrgica, relacionada a fatores como inexperiência do cirurgião, hemostasia deficiente, presença de espaço morto ou tensão excessiva na pele podem ter colaborado para esta ocorrência (Greene \& Dearmin 2006, Fossum \& Willard 2007). Na classe de cirurgias limpa-contaminadas, o valor de 4,25\% de ISC está abaixo dos valores encontrados em literatura, mas nesta classe de pacientes $2,38 \%$ do total de animais apresentou infecção da corrente sanguínea. Nos dois casos 


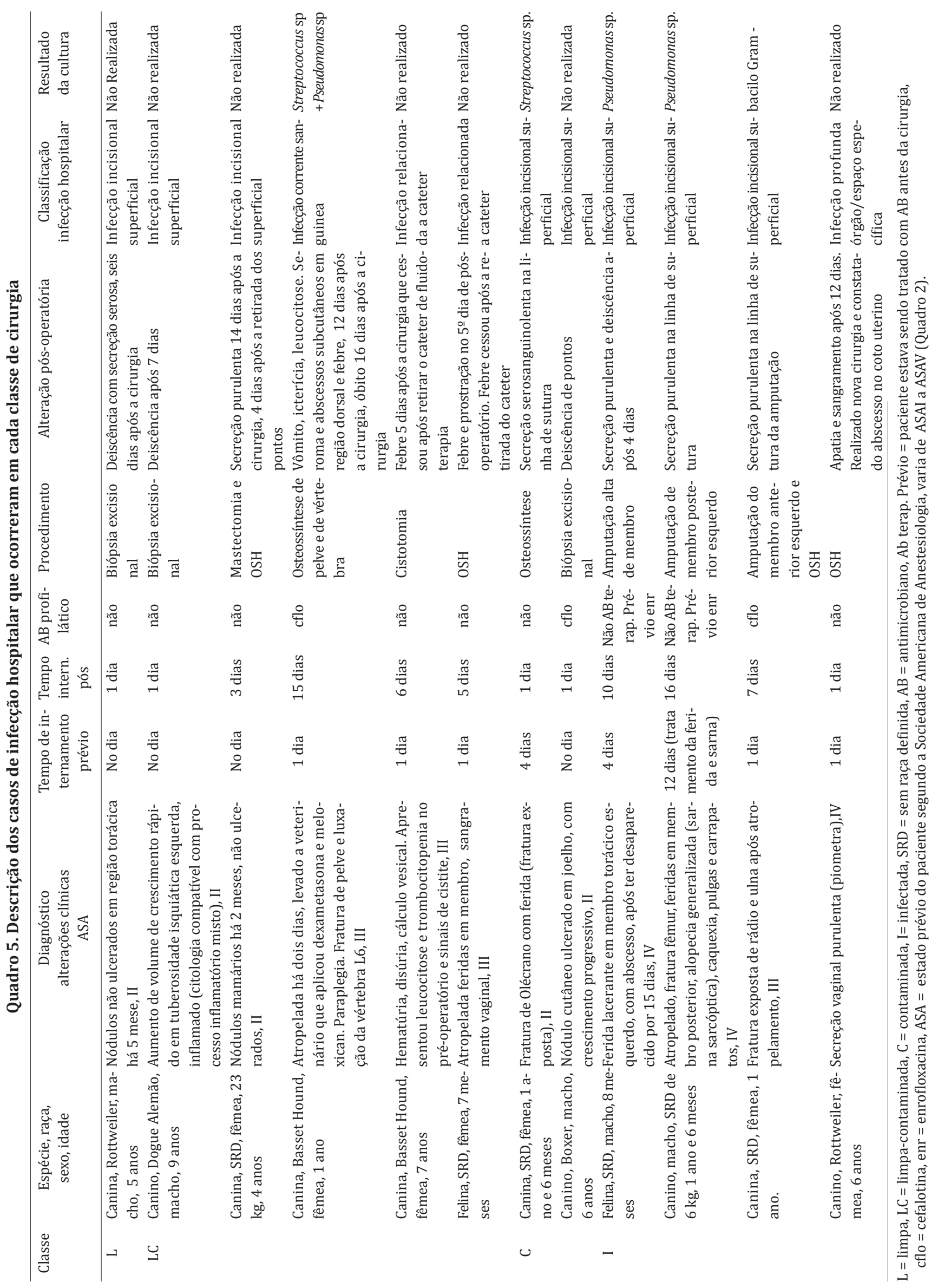


Quadro 6. Resultado do antibiograma com as cepas isoladas dos animais infectados, divididos de acordo com a classificação da cirurgia ou procedimento

\begin{tabular}{|c|c|c|c|c|c|c|c|c|c|c|c|c|c|c|}
\hline $\begin{array}{c}\text { Classe ou } \\
\text { procedimento }\end{array}$ & $\begin{array}{c}\text { Espécie } \\
\text { bacteriana }\end{array}$ & Amic & Gen & Amx & $A m x+a c$ & Amp & Cfl & Clin & Enr & Cipr & Nor & Sul & $\begin{array}{l}\text { Resistência do micro- } \\
\text { organismo por total de } \\
\text { antimicrobiano testados }\end{array}$ & $\begin{array}{l}\text { Resistência do micro- } \\
\text { organismo por classe } \\
\text { de antimicrobiano }\end{array}$ \\
\hline \multirow[t]{2}{*}{$\mathrm{LC}$} & Streptococcus sp. & - & - & - & $\mathrm{S}$ & S & $\mathrm{R}$ & - & $\mathrm{R}$ & $\mathrm{R}$ & $\mathrm{R}$ & - & $4 / 6$ & $2 / 3$ \\
\hline & Pseudomonas sp. & $\mathrm{R}$ & $\mathrm{S}$ & - & $\mathrm{R}$ & $\mathrm{R}$ & $\mathrm{R}$ & - & $\mathrm{R}$ & $\mathrm{S}$ & $\mathrm{S}$ & - & $5 / 8$ & $4 / 4$ \\
\hline $\mathrm{C}$ & Streptococcus sp. & - & $\mathrm{R}$ & - & $\mathrm{S}$ & - & $\mathrm{R}$ & - & $\mathrm{S}$ & $\mathrm{R}$ & $\mathrm{R}$ & $\mathrm{R}$ & $5 / 7$ & $4 / 4$ \\
\hline \multirow[t]{3}{*}{ I } & Pseudomonas sp. & $\mathrm{R}$ & $\mathrm{R}$ & $\mathrm{R}$ & - & - & $\mathrm{R}$ & - & $\mathrm{S}$ & S & & $\mathrm{R}$ & $5 / 7$ & $4 / 5$ \\
\hline & Pseudomonas sp. & - & S & - & $\mathrm{R}$ & - & $\mathrm{R}$ & - & $\mathrm{R}$ & $\mathrm{S}$ & S & $\mathrm{R}$ & $3 / 7$ & $4 / 5$ \\
\hline & Bacilo Gram - & - & $\mathrm{R}$ & $\mathrm{R}$ & - & - & $\mathrm{R}$ & $\mathrm{R}$ & $\mathrm{S}$ & - & & $\mathrm{S}$ & $4 / 6$ & $4 / 6$ \\
\hline Sondagem vesical & Acinetobacter sp.* & $\mathrm{R}$ & $\mathrm{R}$ & & $\mathrm{R}$ & $\mathrm{R}$ & $\mathrm{R}$ & & $\mathrm{R}$ & $\mathrm{R}$ & $\mathrm{R}$ & $\mathrm{R}$ & $9 / 9$ & $5 / 5$ \\
\hline
\end{tabular}

$\begin{array}{lllllllllll}\text { Total resistência por antimicrobiano } & 3 / 3 & 4 / 6 & 2 / 2 & 3 / 5 & 2 / 3 & 7 / 7 & 1 / 1 & 4 / 7 & 3 / 3 & 3 / 5\end{array}$

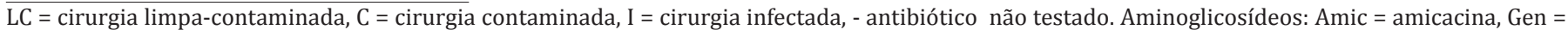
gentamicina. Penicilinas: Amx = amoxacilina, Amox+ac = amoxacilina + ácido clavulânico, Amp = ampicilina. Cefalosporinas: Cfl = cefalexina. Lincosamidas: Clin= clindamicina. Quinolonas: Enr: enrofloxacina, Cipr = ciprofloxacina, Nor = norfloxacina. Sulfonamidas: Sul = sulfazotrim.

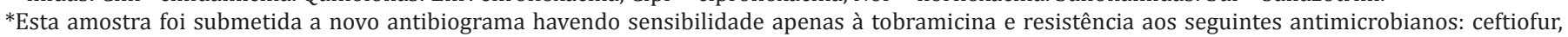
cefoperazone, cefuroxima, danofloxacina, nitrofurantoína, oxacilina e orbifloxacin.

que apresentaram sinais de infecção incisional superficial não foram realizadas culturas microbiológicas, dificultando a confirmação das suspeitas. No caso do paciente com polifraturas em pelve e fratura em vértebra lombar, os procedimentos de apoio, como antibioticoterapia, uso de cateter venoso e sonda vesical foram intensamente utilizados. Após 12 dias detectaram-se sinais de sepse e apesar de todas as medidas, o paciente veio a óbito. Na necropsia foram encontrados abscessos difusos em tecido subcutâneo, sem sinais de contaminação do local cirúrgico, havendo pielonefrite sem cistite, indicando provável infecção de origem endógena, favorecida pela gravidade das lesões, uso de antimicrobianos, intensidade dos procedimentos invasivos (Fernandes et al. 2000) e provável imunossupressão causada pela aplicação prévia de dexametasona (Fernandes et al. 2000, Greene \& Dearmin 2006). Neste caso isolaram-se dos abscessos e da urina duas bactérias multiresistentes, Pseudomonas sp. e Streptococcus sp.

Na classe de cirurgias contaminadas, $10,53 \%$ dos pacientes apresentou infecção incisional superficial, o que está dentro dos valores recomendados. Um canino com fratura de olécrano exposta apresentou secreção serosanguinolenta na linha de sutura, havendo crescimento de Streptococcus sp. multirresistente. Devido à secreção e o crescimento bacteriano, os sinais foram compatíveis com infecção incisional superficial. Na presença de implantes, o aparecimento de sinais até um ano após a cirurgia é compatível com infecção do sítio cirúrgico, devendo-se monitorar estes pacientes por longo prazo (Kirby \& Mazuski 2009).

Na classe de cirurgias infectadas, uma cadela apresentou 12 dias após a OSH sinais clínicos que levaram à realização de uma nova celiotomia, constatando-se ISC do tipo órgão/cavidade (Fernandes et al. 2000, Brasil 2009, Kirby \& Mazuski 2009). Os elementos básicos para a ocorrência da infecção provavelmente foram o reservatório, neste caso o útero infectado, a quantidade do agente inoculado e sua virulência, além da baixa capacidade de defesa do hospedeiro (Fernandes et al. 2000). Três pacientes submetidos à amputação apresentaram sinais de infecção incisional superficial. Dois desses animais permaneceram internados por longo período para permitir a melhora de suas condições clínicas até o momento da cirurgia o que pode ter favorecido a colonização da região inguinal por Pseudomonas sp. Organismos Gram-negativos colonizam certas áreas do corpo, como axilas, períneo e virilha e pacientes com incisão nestas áreas podem apresentar infecção do sítio cirúrgico por este tipo de organismo (Kirby \& Mazuski 2009), conforme observado nos três casos de amputação.

Em medicina humana, o índice de infecção pós-cirúrgica descrita varia entre $1,5 \%$ a $5,1 \%$ nas cirurgias limpas, 7,7 a $10,8 \%$ nas limpas contaminadas, 15,2 a $16,3 \%$ nas contaminadas e 28 a $40 \%$ nas cirurgias infectadas (Lacerda 1992). Em veterinária há citação de taxas globais de ISC de 2 a 40\% (Brown et al. 1997, Dunning 2003, Eugster 2004, Weigelt 2004; Greene \& Dearmin 2006, Fossum \& Willard 2007), supondo que técnicas assépticas, profilaxia antimicrobiana e manejo adequado tenham sido realizados (Eugster 2004, Weigelt 2004). Segundo Brown (2012), o sistema de divisão de classes de ferida cirúrgica foi feito com base no grau de contaminação esperado, havendo muitas variáveis e fatores afetando a cicatrização e taxas de infecção, como a duração da cirurgia, da anestesia e condição do paciente. Apesar da maior parte dos pacientes não apresentar sinais sistêmicos no momento da cirurgia, mais da metade dos casos de infecção ocorreu em pacientes ASA III ou IV, que sofreram trauma prévio ou apresentavam infecção prévia, corroborando com os fatores descritos acima (Brown 2012).

Constatou-se que a profilaxia antimicrobiana, isto é, a administração de antimicrobianos no momento da indução anestésica, em geral uma hora a 30 minutos antes da incisão cirúrgica, um procedimento recomendado nas cirurgias limpa-contaminadas e contaminadas (Johnson 2002, Brown 2012), não foi realizada de maneira adequada pela falta de padronização deste procedimento na instituição. Por outro lado, antimicrobianos foram usados por vários dias em todos os pacientes após a cirurgia, mesmo quando não recomendado, como no caso de cirurgias limpas (Brown 2012). Após os resultados observados no presente trabalho, tem sido ministradas aulas de educação continuada anualmente sobre o assunto antibioticoterapia profilática e terapêutica visando corrigir o problema, e o antibiótico 
profilático foi alterado para cefazolina conforme preconizado por Dunning 2003 e Weigelt 2004. A exposição a antimicrobianos altera a microbiota do paciente predispondo-o a colonização e posterior infecção com bactérias multirresistentes, principalmente se o animal está debilitado ou é submetido a diversos procedimentos invasivos (Johnson 2002, Ogeer-Gyles et al. 2006, Brown 2012). A profilaxia antimicrobiana iniciada antes da cirurgia deve ser repetida a cada duas ou três horas durante a cirurgia, não havendo benefício em mantê-la após 24 horas, a não ser que haja a suspeita de infecção e, neste caso, indica-se um antimicrobiano diferente do utilizado na profilaxia, com fins terapêuticos (Johnson 2002).

Dois pacientes apresentaram sinais de infecção relacionada ao cateter. Segundo Greene (2006), o uso impróprio dos cateteres intravenosos resulta em alta prevalência de infecções nosocomiais. Esta infecção pode ter origem da contaminação do produto, da pele do paciente, ou mais comumente das mãos das pessoas que inserem o cateter. Embora a cultura não tenha sido realizada nos casos aqui descritos, a melhora do quadro após a retirada do cateter é fortemente indicativa de infecção, reforçando a necessidade de realização de treinamento nas técnicas de lavagem de mãos, antissepsia da pele do paciente quando da colocação de cateteres, além de cuidados adequados na manipulação dos mesmos (Lacerda 1992, Johnson 2002, Ogeer-Gyles 2006). Segundo Greene \& Dearmin (2006), além do evento traumático que gera a hospitalização, cateteres intravenosos e urinários são uma das causas mais comuns de infecção hospitalar e pacientes com cateterização endovenosa prolongada devem ser monitorados quanto a infecções, devendo ser realizada a troca periódica dos cateteres pelo menos a cada 72 horas (Greene 2006).

No felino que apresentou infecção urinária após desobstrução uretral e necessitou permanecer sondado por cinco dias, houve crescimento de Acinetobacter sp., bactéria Gram-negativa reconhecida como patógeno nosocomial em humanos desde 1970 (Francey 2000). É encontrado como membro da microbiota da pele e de mucosas de humanos e animais (Bergogne-Bérézin \& Tower 1996), mas também é conhecido como um patógeno oportunista responsável por infecções nosocomiais com considerável morbidade e mortalidade em hospitais (Greene 2006), apresentando taxa de infecção de 0,3\% em pacientes criticamente doentes (Francey 2000). A maioria das infecções do trato urinário está associada ao uso dos cateteres urinários (Johnson 2002), pois a presença da sonda prejudica a barreira exercida pelo fluxo urinário permitindo a entrada de micro-organismos (Fernandes et al. 2000, Johnson 2002). A administração profilática de antimicrobianos não reduz a ocorrência de infecção, ao contrário, aumenta o risco de infecções com bactérias resistentes como constatado no paciente em questão, que havia recebido enrofloxacina por cinco dias durante a permanência da sonda uretral, sendo que a incidência de infecção aumenta 5 a $7 \%$ a cada dia de cateterização (Johnson 2002).

Como constatado neste trabalho, a infecção hospitalar em pacientes cirúrgicos e não cirúrgicos é uma realidade em veterinária, sendo relacionada a vários fatores. Tem grande importância não só devido ao impacto direto sobre os pacientes, mas também devido ao fato de que bactérias multirresistentes podem ser transmitidas de animais domésticos à pessoas (Johnson 2002, Ogeer-Gyles et al. 2006) e vice-versa, o que atualmente pode ser facilitado pelo contato mais próximo que essas espécies mantém (Umber \& Bender 2009). Vários fatores favoreceram a ocorrência de infecção, devendo-se adotar condutas na prevenção da infecção hospitalar em veterinária (Johnson 2002) à semelhança do que é feito em medicina humana (Ogeer-Gyles et al. 2006, CDC 2008, Brasil 2009).

\section{CONCLUSÕES}

O índice de ISC foi de 7,96\% sendo 4,54\% nas cirurgias limpas, 4,25\% nas cirurgias limpa-contaminadas, $10,53 \%$ nas cirurgias contaminadas e $16 \%$ nas cirurgias infectadas.

A taxa de infecção não cirúrgica no paciente cirúrgico foi de 2,88\% e 3,7\% no paciente não cirúrgico.

Foram cultivados sete cepas bacterianas, sendo Pseudomonas sp. (3), Streptococcus sp. (2), Acinetobacter sp. (1) e bacilo Gram-negativo (1), constatando-se multirresistência bacteriana alta em todos as cepas.

A duração da cirurgia e os tempos de internamento pré e pós-operatório não influenciaram na ocorrência de infecção hospitalar, mas os fatores que colaboraram para a ocorrência de infecções no presente trabalho foram a própria gravidade da doença que motivou o tratamento e o tipo de procedimento realizado.

\section{REFERÊNCIAS}

Andrade M.A., Mesquita A.J., Silva L.A.F. \& Paulo N.M. 1992. Frequência de bactérias isoladas no ambiente, em feridas cirúrgicas, em médicos veterinários, enfermeiros e auxiliares de enfermagem. I. Infecção em Hospital Veterinário. Anais Esc. Agron. Vet. 21/22:1-11.

Bergogne-Bérézin E. \& Towner K.J. 1996. Acinetobacter sp. as nosocomial pathogens: Microbiological clinical and epidemiological features. Clin. Microbiol. Rev. 9:148-165.

Bernis Filho W.O., Rezende C.M.F., Abreu V.L.V. \& Bernis V.M.O. 1998. Infecções hospitalares em feridas cirúrgicas de pequenos animais. Arq. Bras. Med. Vet. Zootec. 50:127-132.

Braga D.P. 2008. Incidência e fatores de risco associados à infecção do sítio cirúrgico na clínica de cães e gatos do hospital veterinário da Universidade Federal de Viçosa. Dissertação de Mestrado em Medicina Veterinária, Universidade Federal de Viçosa, Viçosa, MG. 104p.

Brasil. Ministério da Saúde. 2009. Sítio Cirúrgico. Critérios Nacionais de Infecções relacionadas à assistência à saúde: Available at <http://www. infectologia.org.br/anexos/Manual\%20Anvisa_Critérios\%20Nacionais\%20ISC.pdf> Acesso em 25 mai. 2009.

Brown D.C., Conzemius M.G., Shofer F. \& Swann H. 1997. Epidemiological evaluation of postoperative wound infection in dogs and cats. J. Am. Vet. Med. Assoc. 210(9):1302-1306.

Brown D.C. 2012. Wound infection and antimicrobial use, p.135-139. In: Tobias K.M. \& Johnston S.A. (Eds), Veterinary Surgery, Small Animal. Elsevier Saunders, Missouri.

Centers for Disease Control and Prevention 2008. National Healthcare Safety Network (NHSN) Manual. Atlanta. 98p. Available at <http://www. cdc.gov/ncidod/dhqp/pdf/nhsn/NHSN_Manual_PatientSafetyProtocol_CURRENT.pdf $>$ Acesso em jul. 2008.

CLSI 2008. Performance standards for antimicrobial disk and dilution susceptibility tests for bacteria isolated from animals. $3^{\text {rd }}$ ed. Approved Standard M31-A3, Clinical and Laboratory Standards Institute, Wayne, PA. 
Costa E.O., Benites N.R., Tong P., Ferreira M.A., Safatle A.M.V., Baccarin R.Y.A. \& Bellenzani M.C.R. 1996. Avaliação da microbiota aeróbica em centros cirúrgicos médico-veterinários. Anais XXIV Congresso Brasileiro de Medicina Veterinária, Goiânia, GO. p.40. (Resumo)

Dunning D. 2003. Surgical wound infection and the use of antimicrobials, p.113-122. In: Slatter D. (Ed.), Textbook of Small Animal Surgery. Saunders-Elsevier, St Louis.

Eugster S., Schwawalder P., Gaschen F. \& Boerlin P.A. 2004. Prospective study of postoperative surgical site infections in dogs and cats. Vet. Surg. 33:542-550.

Fernandes A.T., Fernandes M.O.V. \& Filho N.R. 2000. Infecção Hospitalar e suas Interfaces na Área de Saúde. Atheneu, São Paulo. 953p.

Fossum T.W. \& Willard M.D. 2007. Surgical infection and antibiotic selection, p.79-89. In: Fossum T.W. (Ed.), Small Animal Surgery. $3^{\text {rd }}$ ed. Mosby Elsevier, Missouri.

Francey T., Gaschen F., Nicolet J. \& Burnens A.P. 2000. The role of Acinetobacter baumanni as a nosocomial pathogen for dogs and cats in an Intensive Care Unit. J. Vet. Intern. Med. 14:177-183.

Greene C.E. \& Dearmin M.G. 2006. Surgical and traumatic wound infections, p.524-531. In: Greene C.E. (Ed.), Infectious Diseases of the Dog and Cat. $3^{\text {rd }}$ ed. Saunders-Elsevier, St Louis.

Greene C.E. 2006. Environmental factors in infectious diseases, p.9911013. In: Ibid. (Ed.), Infectious Diseases of the Dog and Cat. $3^{\text {rd }}$ ed. Saunders-Elsevier, St Louis.

Johnson J.A. 2002. Nosocomial infections. Vet. Clin. North Am., Small Anim. Pract. 32(5):1101-1126.

Kirby J.P. \& Mazuski J.E. 2009. Prevention of surgical site infection. Surg. Clin. North Am. 89:365-389.

Lacerda R.A. 1992. Buscando Compreender a Infecção Hospitalar no Paciente Cirúrgico. Atheneu, São Paulo. 178p.

Mangram A.J., Horan T.C., Pearson M.L., Silver L.C. \& Jarvis W.R. 1999. Guideline for prevention of surgical site infection. Am. J. Infect. Control. 20:247-278

Marsh N.G.M.L., Burney D.P. \& Garcia J. 2007. Surveillance of infections associated with intravenous catheters in dogs and cats in an extensive care unit. J. Am. Anim. Hosp. Assoc. 43:13-20.

Mendes R.C., Arrué L., Mattei A., Antunes T., Nobre M., Ribeiro G. \& Coimbra H. 2005. Presença de Staphylococcus sp. em ambientes de clínicas veterinárias em Pelotas, RS. XIV Congresso de Iniciação Científica da UFPEL. Available at <http://www.ufpel.edu.br/cic/2005/arquivos/conteudo_ CA.html\#00431>

Nicholson M., Beal M., Shofer F. \& Brown D.C. 2002. Epidemiologic evaluation of postoperative wound infection in clean-contaminated wounds: a retrospective study of 239 dogs and cats. Vet. Surg. 31:577-581.

Ogeer-Gyles J.S., Mathews K.A. \& Boerlin P. 2006. Nosocomial infections and antimicrobial resistence in critical care medicine. J. Vet. Crit. Care. 16:1-18,

Pelligand L., Hammond R. \& Rycroft A. 2007. An investigation of the bacterial contamination of small animal breathing systems during routine use. Vet. Anaesthes. Analg. 34:190-199.

Sanchez S., Stevenson M.A., Hudson C.R., Maier M., Buffington T., Dam Q. \& Maurer J.J. 2002. Characterization of multi-drug resistant Escherichia coli isolates associated with nosocomial infections in dogs. J. Clin. Microbiol. 40:3586-3595.

Santos L.R., Neto J.F.S., Rizzo N.N., Bastiani P.V., Rodrigues L.B., Barcellos H.H.A. \& Brum M.V. 2010. Contaminação ambiental em um hospital veterinário e perfil de susceptibilidade a antimicrobianos das bactérias isoladas. Ciênc. Anim. Bras. 11:384-389.

Santos W.G., Diniz R.C., Carvalho I.A. \& Freitas P.M.C.F. 2012. Infecção hospitalar em medicina veterinária. Revta Vet. Zootec. Minas 21:10-15.

Umber J.K. \& Bender J.B. 2009. Pets and antimicrobial resistance. Vet. Clin. Small Anim. 39:279-292.

Weese J.S. 2012 Monitoring for surgical infection, p.170-179. In: Tobias K.M. \& Johnston S.A. (Eds), Veterinary Surgery, Small Animals. Elsevier Saunders, Missouri.

Weigelt J. 2004. Appropriate antimicrobial profile for surgical prophylaxis. Proc. International Veterinary Emergency and Critical Care Symposium, San Diego, California. <http://www.vin.com/Members/Proceedings/ Proceedings.plx?CID=iveccs2004\&PID=pr07186\&O=VIN> 